\title{
Fluctuation Results For Quadratic Continuous-State Branching Process
}

\author{
Hongwei Bi \\ University of international business and economics, 100029, Beijing, China. \\ Email: bihw2009@gmail.com
}

\begin{abstract}
In this note, some fluctuation results for renormalized number of ancestors of a stationary quadratic continuous-state branching process are given. We consider three different cases: same time, different step width; different time, the same step width; adjacent time with the same step width. The Laplace transform of some related quantities is derived to prove this result.
\end{abstract}

Key words and phrases. Fluctuation, stationary CB process, number of ancestor.

2010 Mathematical Subject Classification.60J80,60J85.

\section{Introduction}

Continuous state branching processes (CB processes) are non-negative real-valued Markov processes first introduced by Jirina [4] to model the evolution of large populations of small particles. Continuous state branching processes with immigration (CBI processes) are generalizations of those describing the situation where immigrants may come from outer sources, see e.g. Kawazu and Watanabe [5]. For a survey in this direction, you may refer to Li [7] and the reference therein. Since then they have been powerful tools in biology. In their seminar work, Chen and Delmas [2], stationary continuous-state branching process is considered and they use the immortal decomposition to calculate some quantities associated to the most recent common ancestor (MRCA) and the number of ancestor. They also consider the quadratic CB process as an example and then the renormalized fluctuation result for the number of ancestor is partially considered, and some interesting phenomenon is revealed. In this note, we are going to consider some fluctuation results associated to $\mathrm{M}_{t}^{S}$ (to be specified later) for fixed time $s$ in Section 2. To state our main result, we willprovide in the introduction some background in the following subsections. In particular, wefirst recall the definition of CB process in Subsection 1.1, and then the tree formulation in Subsection1.2, the stationary CB process and the ancestor process associated to stationary CB process are respectively introduced in Subsection 1.3 and 1.4 .

1.1. Quadratic CB process. Consider a sub-critical branching mechanism

$$
\psi(\lambda)=\beta \lambda^{2}+2 \beta \theta \lambda
$$

with fixed $\theta, \beta>0$. Let $\mathbb{P}_{x}$ be the law of a CB $Y=\left(Y_{t}, t \geq 0\right)$ started at mass $x$ with branching mechanism $\psi$. We extend $Y$ on $\mathbb{R}$ by setting $Y_{t}=0$ for $t<0$. Let $\mathbb{E}_{x}$ and $\mathbb{N}$ be respectively the expectation and the canonical measure (excursion measure) associated to $Y$. For every $t>0$ and $\lambda>-\frac{2 \theta}{1-\mathrm{e}^{-2 \beta \theta t}}$, we have

$$
\mathbb{E}_{x}\left[\mathrm{e}^{-\lambda Y_{t}}\right]=\mathrm{e}^{-x u(\lambda, t)}
$$

with

$$
u(\lambda, t)=\mathbb{N}\left[1-\mathrm{e}^{-\lambda Y_{t}}\right]=2 \theta \lambda\left[(2 \theta+\lambda) \mathrm{e}^{2 \beta \theta t}-\lambda\right]^{-1} .
$$

Then it is easy to derive that for $t>0$,

$$
\beta \int_{0}^{t} u(\lambda, r) d r=\log \left(1+\lambda \frac{1-\mathrm{e}^{-2 \beta \theta t}}{2 \theta}\right) \quad \text { and } \beta \int_{0}^{\infty} u(\lambda, t) d t=\log \left(1+\frac{\lambda}{2 \theta}\right) .
$$

Let $c(t)=\lim _{\lambda \rightarrow \infty} u(\lambda, t)$ and denote by $\zeta=\inf \left\{t>0 ; Y_{t}=0\right\}$ the lifetime of $Y$ under $\mathbb{N}$. Then we have

$$
c(t)=\mathbb{N}[\zeta>t]=2 \theta\left(\mathrm{e}^{2 \beta \theta t}-1\right)^{-1} .
$$


1.2. The real tree formulation. Define for $r>0, \mathbf{M}_{-r}^{0}$, the number of ancestors (the immortal individual excluded) at time $-r$ of the current population at time 0 . In order to define precisely this quantity, some finer structure for $\mathrm{CB}$ process is needed. We recall in what follows the genealogical tree for the CB process which is studied in Le Gall [6] or Duquesne and Le Gall [3]. Since the branching mechanism is quadratic, the corresponding Levy process is just the Brownian motion with drift. Let $B=\left(B_{t}, t \in R_{+}\right)$be a standard Brownian motion. We consider the Brownian motion $\mathrm{B}^{\theta}=\left(B_{t}^{\theta}, t \in R_{+}\right)$with negative drift and the corresponding reflected process above its minimum $H=\left(H(t), t \in R_{+}\right)$:

$$
B_{t}^{\theta}=\sqrt{\frac{2}{\beta}} B_{t}-2 \theta t \quad \text { and } \quad H(t)=B_{t}^{\theta}-\inf _{s \in[0, t]} B_{s}^{\theta} .
$$

We deduce from equation (1.7) in [3] that $H$ is the height process associated to the branching mechanism $\psi$. For a function $H$, set $\max (H)=\max \left(H(t), t \in R_{+}\right)$. Let $\mathrm{N}[d H]$ be the excursion measure of $H$ above Onormalized such that $\mathrm{N}[\max (H) \geq r]=c(r)$. Let $\left(l_{t}^{x}(H), x \in R_{+}, t \in R_{+}\right)$be the local time of $H$ at time $t$ and level $x$. Let $\zeta=\inf \{t>0 ; H(t)=0\}$ be the duration of the excursion $H$ under $\mathrm{N}[d H]$. We recall that $\left(l_{\zeta}^{r}(H), r \in R_{+}\right)$under $\mathrm{N}$ is distributed as $Y$ under $\mathbb{N}$. From now on we shall identify $Y$ with $\left(l_{\zeta}^{r}(H), r \in R_{+}\right)$and write $\mathbb{N}$ for $\mathrm{N}$. We now recall the construction of the genealogical tree of the CB process $Y$ from $H$.

Let $f$ be a continuous non-negative function defined on $[0,+\infty)$ with compact support, such that $f(0)=0$. We set $\zeta^{f}=\sup \{t ; f(t)>0\}$, with the convention that $\sup \emptyset=0$. Let $d^{f}$ be the non-negative function defined by:

$$
d^{f}(s, t)=f(s)+f(t)-2 \inf _{u \in[s \wedge t, s \vee t]} f(u) .
$$

It can be easily checked that $d^{f}$ is a semi-metric on $\left[0, \zeta^{f}\right]$. One can define the equivalence relation associated to $d^{f}$ by $s \sim t$ if and only if $d^{f}(s, t)=0$. Moreover, when we consider the quotient space $T^{f}=\left[0, \zeta^{f}\right] / \sim$ and, noting again $d^{f}$ the induced metric on $T^{f}$ and rooting $T^{f}$ at $\emptyset_{f}^{f}$. the equivalence class of 0 . it can be checked that the space, $\left(T^{f}, \cdot d^{f}, \emptyset^{f},\right)$ is a compact rooted real tree. The so-called genealogical tree of the CB process $Y$ is the real tree $\mathcal{T}=\left(T^{H}, d^{H}, \emptyset^{H}\right)$. In what follows, we shall mainly present the result using the height process $H$ instead of the genealogical tree $\mathcal{T}$, and say that $H$ codes for the genealogy of $Y$.

Let $a>0$ and $\left(H_{k}, k \in \mathcal{K}_{a}\right)$ be the excursions of $H$ above level $a$. It is well known that $\sum_{k \in \mathcal{K}_{a}} \delta_{H_{k}}(d H)$ is under $\mathbb{N}$ and conditionally on $\left(Y_{r}, r \in[0, a]\right)$, a Poisson point measure with intensity $Y_{a} \mathbb{N}[d H]$. We define the number of ancestors at time $a$ of the population living at time $b$ as the number of excursions above level $a$ which reach level $b>a$ by:

$$
R_{a}^{b}(H)=\sum_{k \in \mathcal{K}_{a}} \mathbf{1}_{\left\{\max \left(H_{k}\right) \geq b-a\right\}} .
$$

When there is no risk of confusion, we shall write $R_{a}^{b}$ for $R_{a}^{b}(H)$.

1.3. Stationary CB process. Let $\mathrm{D}$ be the space of cadlag paths having 0 as a trap. Consider underP a Poisson point measure

$$
\mathcal{N}(d t, d Y)=\sum_{i \in I} \delta_{t_{i}, Y_{i}}(d t, d Y)
$$

on $\mathbb{R} \times \mathbb{D}$ with intensity $2 \beta d t \mathbb{N}[d Y]$. We shall consider the process $Z=\left(Z_{t}, t \in \mathbb{R}\right)$ defined by

$$
Z_{t}=\sum_{t_{i} \leq t} Y_{t-t_{i}}^{i}
$$

Let $\mathrm{E}$ be the corresponding expectation. Forwarding to [2], $\mathrm{Z}$ is a $\mathrm{CB}$ process conditionally on non-extinction and moreover a stationary $\mathrm{CB}$ process (Certainly it can be treated as a $\mathrm{CB}$ process with immigration from $\mathrm{Li}$ [8]). Using the property of the Poisson point measure, we have: 


$$
\mathrm{E}\left[\mathrm{e}^{-\lambda Z_{t}}\right]=\left(1+\frac{\lambda}{2 \theta}\right)^{-2}
$$

In particular,

$$
\mathrm{E}\left[Z_{t}\right]=\frac{1}{\theta} \quad \text { and } \quad \mathrm{E}\left[Z_{t}^{2}\right]=\frac{3}{2 \theta^{2}}
$$

\subsection{The ancestor process.Let}

$$
\mathcal{N}^{\prime}(d t, d H)=\sum_{i \in I} \delta_{\left(t_{i}, H^{i}\right)}(d t, d H)
$$

be a Poisson point measure with intensity $2 \beta d t \mathbb{N}[\mathrm{dH}]$. We will write $Y_{a}^{i}$ for $l^{a}\left(H^{i}\right)$ for $i \in I$. Thus $\sum_{i \in I} \delta_{\left(t_{i}, H^{i}\right)}$ allows to code (on an enlarged space) the genealogy of $Z$ defined by (5).

Let $r<$ t. Define explicitly the number of ancestors (excluding the immortal particle) at time $r$ of the population living at timet, $M_{r}^{t}$, by

$$
M_{r}^{t}=\sum_{i \in I} \mathbf{1}_{\left\{t_{i}<r\right\}} R_{r-t_{i}}^{t-t_{i}}\left(H^{i}\right) .
$$

We will always identify $M_{-r}$ with $M_{-r}^{0}$ when there is no risk of confusion. Notice that for $r$ larger than the time to most recent common ancestor (TMRCA) we have $M_{-r}=0$. It is easy to deduce the following identities.

$$
\begin{gathered}
\mathrm{E}\left[M_{-t}\right]=\frac{c(t)}{\theta}, \quad \mathrm{E}\left[M_{-t}^{2}\right]=\frac{c(t)}{\theta}\left(1+\frac{3}{2} \frac{c(t)}{\theta}\right)=2 \frac{\mathrm{e}^{2 \beta \theta t}+2}{\left(\mathrm{e}^{2 \beta \theta t}-1\right)^{2}}, \\
\mathrm{E}\left[\mathrm{e}^{-\lambda M_{-r}}\right]=\mathrm{E}\left[\mathrm{e}^{-\left(1-\mathrm{e}^{-\lambda}\right) c(r) Z_{-r}}\right]=\left(1+\frac{c(r)}{2 \theta}\left(1-\mathrm{e}^{-\lambda}\right)\right)^{-2},
\end{gathered}
$$

The next result is in a sense a consequence of the time reversibility of the process $Y$ with respect to its lifetime $\zeta$ which was derived from [1].

Lemma 1.1. $\left(Z_{0},\left(M_{-t}, t>0\right)\right)$ and $\left(Z_{0},\left(M_{0}^{t}, t>0\right)\right)$ have the same distribution.

Note

$$
\mathcal{M}_{r}^{s}=\sqrt{c(r)}\left(Z_{s}-\frac{M_{s-r}^{s}}{c(r)}\right) .
$$

Our first result concerns with the same current time $s$ and different step width (Theorem2.1), that is, conditional on converges in the ognse of finite dimensional to $\left(\sqrt{Z_{s}} U_{t}^{s}, t \geq 0\right)_{\text {with }} U^{s}=\left(U_{t}^{s}, t \geq 0\right)$ a Gaussian process. It is somewhat anti-intuitive.

The second result considers with different current time $s_{i}$ while with the same step width(Theorem 2.2): let $\mu_{i}>0$ and $s_{i} \geq s_{i+1}$ for $1 \leq i \leq n$. Then

$$
\mathrm{E}\left[\exp \left(-\sum_{i=1}^{n} \mu_{i} \mathcal{M}_{r}^{s_{i}}\right)\right] \stackrel{r \downarrow 0}{\longrightarrow} \mathrm{E}\left[\exp \left(\sum_{i=1}^{n} \frac{\mu_{i}^{2}}{2} Z_{s_{i}}\right)\right] .
$$

Subsection 2.2 and 2.3 are devoted to proving the two results using some induction tools.

\section{Main Results On The Number Of Ancestors}

In this section, we focus on the fluctuations on the number of ancestors $M_{t}^{s}$ associated toa fixed time $s$. In particular we shall deal with the fluctuations from three point of view: thesame starting time with different step width; different starting time with same step width;adjacent time with the same step width.

2.1. Main Results. First let us recall some known results from [2]. For $r>0$, we note 


$$
\mathcal{M}_{r}^{s}=\sqrt{c(r)}\left(Z_{s}-\frac{M_{s-r}^{s}}{c(r)}\right)
$$

Then we have:

$$
\begin{aligned}
\mathrm{E}\left[\mathrm{e}^{-\alpha \mathcal{M}_{r}^{0}}\right] & =\exp \left(-2 \beta \int_{0}^{r} u(\alpha \sqrt{c(r)}, t) d t\right) \\
& \times \mathrm{E}\left[\exp \left(-Z_{-r}\left[c(r)\left(1-\mathrm{e}^{\alpha \sqrt{c(r)}}\right)+\mathrm{e}^{\alpha \sqrt{c(r)}} u(\alpha \sqrt{c(r)}, r)\right]\right)\right] .
\end{aligned}
$$

This implies that the one-dimensional convergence holds: for $\alpha>0$,

$$
\mathrm{E}\left[\mathrm{e}^{-\alpha \mathcal{M}_{r}^{0}}\right] \stackrel{s \downarrow 0}{\longrightarrow} \mathrm{E}\left[\mathrm{e}^{\frac{\alpha^{2}}{2} Z_{0}}\right] .
$$

We shall consider the normalized fluctuations for the number of ancestors in finite dimensions. The first main result associated to fixed time $s$ with different step width is as follows:

Theorem 2.1. Conditional on $Z_{s}$, the process $\left(\mathcal{M}_{r t}^{s}, t \geq 0\right)$ converges in the sense of finite dimensional to $\left(\sqrt{Z_{s}} U_{t}^{s}, t \geq 0\right)$ with $U^{s}=\left(U_{t}^{s}, t \geq 0\right)$ a Gaussian process such that for $h, t \geq 0, \mathrm{E}\left[U_{t}^{s} U_{t+h}^{s}\right]=\mathrm{e}^{h / 2}$.

The second type of fluctuation is given as follows:

Theorem 2.2. For $1 \leq i \leq n, \mu_{i}>0$ and $s_{i} \geq s_{i+1}$ with the convention that $s_{n+1}=-\infty$, we have:

$$
\mathrm{E}\left[\exp \left(-\sum_{i=1}^{n} \mu_{i} \mathcal{M}_{r}^{s_{i}}\right)\right] \stackrel{r \downarrow 0}{\longrightarrow} \mathrm{E}\left[\exp \left(\sum_{i=1}^{n} \frac{\mu_{i}^{2}}{2} Z_{s_{i}}\right)\right] .
$$

Remark 2.3. Theorem 2.2 implies the processes $U_{s}$ are independent for different $s$.

Remark 2.4. For the third type of fluctuation on the process $\left(\mathcal{M}_{r}^{\delta r}, 0 \leq \delta \leq 1\right)$, we can only get the twodimensional convergence for this process, that is for $\alpha, \eta \geq 0$,

$$
\mathrm{E}\left[\mathrm{e}^{-\alpha \mathcal{M}_{r}^{0}-\eta \mathcal{M}_{r}^{\delta r}}\right] \stackrel{r \downarrow 0}{\longrightarrow} \mathrm{E}\left[\mathrm{e}^{\left(\alpha^{2}+\eta^{2}+2 \alpha \eta(1-\delta)^{2}\right) \frac{Z_{0}}{2}}\right] .
$$

It is not straightforward to get the finite dimensional convergence.

2.2. Proof of Theorem 2.1. By stationarity, we may fix $s=0$. We first get the Laplacetransform associated to $M$ and $Z_{0}$ in the $n$-dimensional case through induction.

Let $\mathrm{b}<\mathrm{a}, \mathrm{c}$. Recall that $R_{a-b}^{c-b}(H)$ denote the number of individuals (coded by $H$ ) at time $a$ (born at time $b$ ) which is still alive at time $c$. Then we have

Lemma 2.5. For $i=1,2, \cdots, n$, let $\mu_{i}>0$ and $t_{1} \leq \cdots \leq t_{n}$. Then

$$
\begin{aligned}
& \mathrm{E}\left[\exp \left(-\sum_{i=1}^{n} \mu_{i} M_{-r \mathrm{e}^{t_{i}}}-\lambda Z_{0}\right)\right] \\
& =\exp \left(-2 \beta \int_{0}^{r \mathrm{e}^{t_{1}}} u\left(\mu_{0}^{\prime}, t\right) d t\right) \times \exp \left(-2 \beta \int_{0}^{r\left(\mathrm{e}^{t_{2}}-\mathrm{e}^{t_{1}}\right)} u\left(\mu_{1}^{\prime}, t\right) d t\right) \cdots \\
& \times \exp \left(-2 \beta \int_{0}^{r\left(\mathrm{e}^{t_{n}}-\mathrm{e}^{t_{n-1}}\right)} u\left(\mu_{n-1}^{\prime}, t\right) d t\right) \mathrm{E}\left[\mathrm{e}^{-\mu_{n}^{\prime} Z_{0}}\right],
\end{aligned}
$$

where the iteration follows with $\mu_{0}^{\prime}=\lambda$ and for $1 \leq i \leq n$,

$$
\mu_{i}^{\prime}=\left(1-\mathrm{e}^{-\mu_{i}}\right) c\left(r \mathrm{e}^{t_{i}}\right)+\mathrm{e}^{-\mu_{i}} u\left(\mu_{i-1}^{\prime}, r\left(\mathrm{e}^{t_{i}}-\mathrm{e}^{t_{i-1}}\right)\right)
$$

with the convention that $t_{0}=-\infty$.

Proof. For $n=1$, it is straightforward to get by Lemma 1.1 that

$$
\mathrm{E}\left[\mathrm{e}^{-\mu_{1} M_{-r} \mathrm{e}^{t_{1}}-\lambda Z_{0}}\right]=\left(1+\frac{\lambda+c\left(r \mathrm{e}^{t_{1}}\right)\left(1-\mathrm{e}^{-\mu_{1}}\right)}{2 \theta}\right)^{-2} .
$$


An application of (2) yields that

$$
\exp \left(-2 \beta \int_{0}^{r \mathrm{e}^{t_{1}}} u\left(\mu_{0}^{\prime}, t\right) d t\right) \exp \left(-2 \beta \int_{0}^{\infty} u\left(\mu_{1}^{\prime}, t\right) d t\right)=\left(1+\frac{\lambda+c\left(r \mathrm{e}^{t_{1}}\right)\left(1-\mathrm{e}^{-\mu_{1}}\right)}{2 \theta}\right)^{-2} .
$$

Thus the equation is valid for 1-dimensional case.

Suppose now that the equation is valid for $n=j \geq 2$, that is,

$$
\begin{aligned}
& \mathrm{E}\left[\exp \left(-\sum_{i=1}^{j} \mu_{i} M_{-r \mathrm{e}^{t_{i}}}-\lambda Z_{0}\right)\right] \\
& =\exp \left(-2 \beta \int_{0}^{r \mathrm{e}^{t_{1}}} u\left(\mu_{0}^{\prime}, t\right) d t\right) \times \exp \left(-2 \beta \int_{0}^{r\left(\mathrm{e}^{t_{2}}-\mathrm{e}^{t_{1}}\right)} u\left(\mu_{1}^{\prime}, t\right) d t\right) \cdots \\
& \times \exp \left(-2 \beta \int_{0}^{r\left(\mathrm{e}^{t_{j}}-\mathrm{e}^{t_{j-1}}\right)} u\left(\mu_{j-1}^{\prime}, t\right) d t\right) \exp \left(-2 \beta \int_{0}^{\infty} u\left(\mu_{j}^{\prime}, t\right) d t\right) .
\end{aligned}
$$

By decomposition with respect to the birth time, for $n=j+1$, we have:

$$
\begin{aligned}
& \mathrm{E}\left[\exp \left(-\sum_{i=1}^{j+1} \mu_{i} M_{-r \mathrm{e}^{t_{i}}}-\lambda Z_{0}\right)\right] \\
& =\mathrm{E}\left[\exp \left(-\lambda \sum_{-r \mathrm{e}^{t_{1}<t_{k}<0}} Y_{-t_{k}}^{k}\right)\right] \\
& \times \mathrm{E}\left[\exp \left(-\sum_{-r \mathrm{e}^{t_{2}}<t_{k}<-r \mathrm{e}^{t_{1}}}\left(\mu_{1} R_{-r \mathrm{e}^{t_{1}-t_{k}}}^{-t_{k}}\left(H^{k}\right)+\lambda Y_{-t_{k}}^{k}\right)\right)\right] \\
& \times \cdots \times \mathrm{E}\left[\exp \left(-\sum_{-r \mathrm{e}^{t_{j+1}<t_{k}<-r \mathrm{e}^{t_{j}}}}\left(\sum_{l=1}^{j} \mu_{l} R_{-r \mathrm{e}^{t_{l}-t_{k}}}^{-t_{k}}\left(H^{k}\right)+\lambda Y_{-t_{k}}^{k}\right)\right)\right] \\
& \times \mathrm{E}\left[\exp \left(-\sum_{t_{k}<-r \mathrm{e}^{t_{j+1}}}\left(\sum_{l=1}^{j+1} \mu_{l} R_{-r \mathrm{e}^{t_{l}}-t_{k}}^{-t_{k}}\left(H^{k}\right)+\lambda Y_{-t_{k}}^{k}\right)\right)\right] .
\end{aligned}
$$

It is clear that for the first $j$ terms, they appear just as in the $n=j$ case. Besides the integrand in the $(j+1)$-th term is the same as that of the last term in $n=j$ case but with different integrating range. We denote the first $j+$ 1 terms as $F_{j+1}$. Thus we have:

$$
\begin{aligned}
F_{j+1} & =\exp \left(-2 \beta \int_{0}^{r \mathrm{e}^{t_{1}}} u\left(\mu_{0}^{\prime}, t\right) d t\right) \times \exp \left(-2 \beta \int_{0}^{r\left(\mathrm{e}^{t_{2}}-\mathrm{e}^{t_{1}}\right)} u\left(\mu_{1}^{\prime}, t\right) d t\right) \cdots \\
& \times \exp \left(-2 \beta \int_{0}^{r\left(\mathrm{e}^{t_{j}}-\mathrm{e}^{t_{j-1}}\right)} u\left(\mu_{j-1}^{\prime}, t\right) d t\right) \exp \left(-2 \beta \int_{0}^{r\left(\mathrm{e}^{t_{j+1}}-\mathrm{e}^{t_{j}}\right)} u\left(\mu_{j}^{\prime}, t\right) d t\right) .
\end{aligned}
$$

For the last term, we have:

$$
\begin{aligned}
& \mathrm{E}\left[\exp \left(-\sum_{t_{k}<-r \mathrm{e}^{t_{j+1}}}\left(\sum_{l=1}^{j+1} \mu_{l} R_{-r \mathrm{e}^{t_{l}-t_{k}}}^{-t_{k}}\left(H^{k}\right)+\lambda Y_{-t_{k}}^{k}\right)\right)\right] \\
& =\exp \left(-2 \beta \int_{0}^{\infty} d t \mathbb{N}\left[1-\exp \left(-\mu_{j+1} R_{t}^{t+r \mathrm{e}^{t_{j+1}}}(H)-F(\lambda, t, \mu, H)\right)\right]\right),
\end{aligned}
$$

where $F(\lambda, t, \mu, H)=\sum_{l=1}^{j} \mu_{l} R_{t+r \mathrm{e}^{t_{j+1}}-r \mathrm{e}^{t_{l}}}^{t+1}(H)+\lambda Y_{t+r \mathrm{e}^{t_{j+1}}}$. The integrand in equation (11) can be written as 
Moreover we have:

$$
\begin{aligned}
& \mathbb{N}\left[1-\exp \left(-\mu_{j+1} R_{t}^{t+r \mathrm{e}^{t_{j+1}}}(H)-F(\lambda, t, \mu, H)\right)\right] \\
& =\mathbb{N}\left[1-\exp \left(-\sum_{q}\left(\mu_{j+1} 1_{\left\{\zeta_{q} \geq r \mathrm{e}^{t_{j+1}}\right\}}+F\left(\lambda, 0, \mu, H_{q}\right)\right)\right)\right]
\end{aligned}
$$

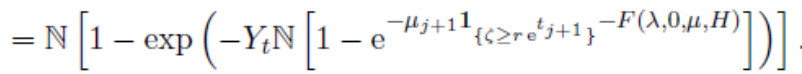

$$
\begin{aligned}
\mathbb{N}\left[1-\mathrm{e}^{-\mu_{j+1} 1_{\left\{\zeta \geq r \mathrm{e}^{\left.t_{j+1}\right\}}\right.}-F(\lambda, 0, \mu, H)}\right] \\
=\left(1-\mathrm{e}^{-\mu_{j+1}}\right) \mathbb{N}\left[\zeta \geq r \mathrm{e}^{t_{j+1}}\right]+\mathrm{e}^{-\mu_{j+1}} \mathbb{N}\left[1-\mathrm{e}^{-F(\lambda, 0, \mu, H)}\right],
\end{aligned}
$$

where it can be deduced from the $n=j$ case that

$$
\mathbb{N}\left[1-\mathrm{e}^{-F(\lambda, 0, \mu, H)}\right]=u\left(\mu_{j}^{\prime}, r\left(\mathrm{e}^{t_{j+1}}-\mathrm{e}^{t_{j}}\right)\right) .
$$

Then we get

as well as

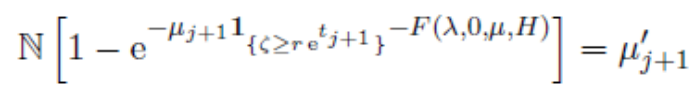

$$
\mathbb{N}\left[1-\mathrm{e}^{-\mu_{j+1} R_{t}^{t+r \mathrm{e}^{t_{j+1}}}(H)-F(\lambda, t, \mu, H)}\right]=u\left(\mu_{j+1}^{\prime}, t\right) .
$$

Thus the equation is valid for $n=j+1$, which completes the proof.

Proof of Theorem 2.1. Let $\mu_{i}=-\frac{\eta_{i}}{\sqrt{c\left(r \mathrm{e}^{\left.t_{i}\right)}\right.}}$ for $1 \leq i \leq n$, and $\lambda=\sum_{i=1}^{n} \eta_{i} \sqrt{c\left(r \mathrm{e}^{t_{i}}\right)}$ in Lemma 2.5 with $\eta_{i} \geq 0$. First it is easy to deduce that as $r \rightarrow 0+$,

$$
u\left(\lambda, r \mathrm{e}^{t_{1}}\right) \sim \lambda-\left(\sum_{i=1}^{n} \eta_{i} \frac{\sqrt{c\left(r \mathrm{e}^{t_{i}}\right)}}{\sqrt{c\left(r \mathrm{e}^{t_{1}}\right)}}\right)^{2} \sim \lambda-\left(\sum_{i=1}^{n} \eta_{i} \mathrm{e}^{\left(t_{1}-t_{i}\right) / 2}\right)^{2} .
$$

An application of (2) yields that

$$
\exp \left(-2 \beta \int_{0}^{r \mathrm{e}^{t_{1}}} u\left(\mu_{0}^{\prime}, t\right) d t\right) \rightarrow 1 \text {. }
$$

For $1 \leq i \leq n-1$, it is elementary to deduce that as $r \rightarrow 0+$,

$$
\mu_{i}^{\prime} \sim \sum_{j \geq i+1} \eta_{j} \sqrt{c\left(r \mathrm{e}^{t_{j}}\right)}-\sum_{j=1}^{i} \frac{\eta_{j}^{2}}{2}+\sum_{k=1}^{i}\left[\sum_{j \geq k} \eta_{k} \eta_{j} \mathrm{e}^{\left(t_{k}-t_{j}\right) / 2}-\left(\sum_{j \geq k} \eta_{j} \sqrt{\mathrm{e}^{t_{k}-t_{j}}-\mathrm{e}^{t_{k-1}-t_{j}}}\right)^{2}\right] .
$$

This entails for $1 \leq i \leq n-1$ that as $r \rightarrow 0+$,

$$
\exp \left(-2 \beta \int_{0}^{r\left(\mathrm{e}^{t_{i}}-\mathrm{e}^{t_{i-1}}\right)} u\left(\mu_{i-1}^{\prime}, t\right) d t\right) \rightarrow 1 .
$$

For the last term, we yield

$$
\mu_{n}^{\prime} \sim-\sum_{j=1}^{n} \frac{\eta_{j}^{2}}{2}+\sum_{k=1}^{n}\left[\sum_{j \geq k} \eta_{k} \eta_{j} \mathrm{e}^{\left(t_{k}-t_{j}\right) / 2}-\left(\sum_{j \geq k} \eta_{j} \sqrt{\mathrm{e}^{t_{k}-t_{j}}-\mathrm{e}^{t_{k-1}-t_{j}}}\right)^{2}\right] .
$$

Some elementary calculations on the right-hand side of (12) yields

$$
\mu_{n}^{\prime} \sim-\frac{1}{2} \sum_{1 \leq i, j \leq n} \eta_{i} \eta_{j} \mathrm{e}^{\left(t_{i}-t_{j}\right) / 2} .
$$

The result thus follows.

2.3. Proof of Theorem 2.2. Recall in the quadratic case, for $\lambda, t>0$,

$$
u(\lambda, t)=\frac{2 \theta \lambda}{2 \theta \mathrm{e}^{2 \beta \theta t}+\lambda\left(\mathrm{e}^{2 \beta \theta t}-1\right)} .
$$


We can further generalize this to $\lambda \in[-2 \theta,+\infty)$ since for anyt $>0, u(\lambda, t)$ can be definedon $\left[f_{\theta}(t), \infty\right)$ with $f_{\theta}(t)=2 \theta\left(1-\mathrm{e}^{-2 \beta \theta t}\right)^{-1}$, while $f_{\theta}(t) \geq 2 \theta$.

We consider the $n$-dimensional case as $r>0$ small enough, $s_{1}>\cdots>s_{n}$ and note $s_{n+1}=-\infty$. By stationarity and the similar calculations as that of Lemma 2.5 , we have:

Lemma 2.6. For $1 \leq i \leq n, \alpha_{i,} \beta_{i} \geq 0$, we get:

(13)

$$
\left.\mathrm{E}\left[\mathrm{e}^{-\sum_{i=1}^{n}\left(\alpha_{i} M_{s_{i}-r}^{s_{i}}+\beta_{i} Z_{s_{i}}\right)}\right]=\exp (-2 \beta)^{n}\left(\int^{r} u\left(\beta_{i}^{\prime}, t\right) d t+\int^{s_{i}-s_{i+1}-r} u\left(\alpha_{i}^{\prime}, t\right) d t\right)\right) .
$$

where inductively,

$$
\left\{\begin{array}{l}
\beta_{i}^{\prime}=\beta_{1}, \quad i=1 \\
\beta_{i}^{\prime}=\beta_{i}+u\left(\alpha_{i-1}^{\prime}, s_{i-1}-s_{i}-r\right), \quad i \geq 2 ; \\
\alpha_{i}^{\prime}=\left(1-\mathrm{e}^{-\alpha_{i}}\right) c(r)+\mathrm{e}^{-\alpha_{i}} u\left(\beta_{i}^{\prime}, r\right), \quad 1 \leq i \leq n .
\end{array}\right.
$$

Then we are ready to prove Theorem 2.2. The following is dedicated to this.

Proof of Theorem 2.2.Note first that equation (13) can also be generalized to the negative case as illustrated above for $u(\lambda, t)$.

Now let $\alpha_{i}=-\mu_{i} / \sqrt{c(r)}, \beta_{i}=\mu_{i} \sqrt{c(r)}$ for $1 \leq i \leq n$ with the condition that $\sum_{i=1}^{n} \mu_{i}^{2} \leq 4 \theta$. We can get the convergence as $r \rightarrow 0+$ from (13) through approximation.

First we deal with $\alpha_{1}^{\prime}$ and $\beta_{1}^{\prime}$. We havethis yiedd $\left(\mu_{1} \sqrt{c(r)}, r\right) \sim \mu_{1} \sqrt{c(r)}-\mu_{1}^{2}$,

$$
\exp \left[-2 \beta \int_{0}^{r} u\left(\mu_{1} \sqrt{c(r)}, q\right) d q\right]=\exp \left[-\log \frac{\psi\left(\mu_{1} \sqrt{c(r)}\right)}{\psi\left(u\left(\mu_{1} \sqrt{c(r)}, r\right)\right) \mathrm{e}^{2 \beta \theta r}}\right] \stackrel{r \downarrow 0}{\longrightarrow} 1 .
$$

In addition as $r \rightarrow 0$,

$$
\alpha_{1}^{\prime} \sim-\left(\frac{\mu_{1}}{\sqrt{c(r)}}+\frac{\mu_{1}^{2}}{2 c(r)}\right) c(r)+\left(1+\frac{\mu_{1}}{\sqrt{c(r)}}\right)\left(\mu_{1} \sqrt{c(r)}-\mu_{1}^{2}\right) \sim-\frac{\mu_{1}^{2}}{2} .
$$

Thus we have:

$$
\exp \left(-2 \beta \int_{0}^{s_{1}-s_{2}-r} u\left(\alpha_{1}^{\prime}, q\right) d q\right) \stackrel{r \downarrow 0}{\longrightarrow} \exp \left(-2 \beta \int_{0}^{s_{1}-s_{2}} u\left(-\frac{\mu_{1}^{2}}{2}, q\right) d q\right) .
$$

Second for $\beta_{2}^{\prime}$, we have $\beta_{2}^{\prime} \sim \mu_{2} \sqrt{c(r)}+u\left(-\frac{\mu_{1}^{2}}{2}, t-s\right)$, which entails

$$
\exp \left(-2 \beta \int_{0}^{r} u\left(\beta_{2}^{\prime}, q\right) d q\right) \stackrel{r \downarrow 0}{\longrightarrow} 1 .
$$

Similar calculations as that of $\alpha_{1}^{\prime}$ gives $\alpha_{2}^{\prime} \sim-\frac{\mu_{2}^{2}}{2}+u\left(-\frac{\mu_{1}^{2}}{2}, s_{1}-s_{2}\right) \geq-\frac{\mu_{1}^{2}+\mu_{2}^{2}}{2}$.

We can now use the induction to deduce that for $i \geq 3$,

$$
\left\{\begin{array}{l}
\alpha_{i}^{\prime} \sim-\frac{\mu_{i}^{2}}{2}+u\left(\alpha_{i-1}^{\prime}, s_{i-1}-s_{i}\right) ; \\
\beta_{i}^{\prime} \sim \mu_{i} \sqrt{c(r)}+u\left(\alpha_{i-1}^{\prime}, s_{i-1}-s_{i}\right) .
\end{array}\right.
$$

Then $\alpha_{i}^{\prime}$ is negative but bounded from below by $-2 \theta$. This yields for every $i$ that

$$
\exp \left(-2 \beta \int_{0}^{r} u\left(\beta_{i}^{\prime}, q\right) d q\right) \stackrel{r \downarrow 0}{\longrightarrow} 1 .
$$


Then under the condition that $\sum_{i=1}^{n} \mu_{i}^{2} \leq 4 \theta$, we have for $r \rightarrow 0+$ :

$$
\begin{aligned}
& \mathrm{E}\left[\mathrm{e}^{-\mu_{i} \mathcal{M}_{s_{i}-r}^{s_{i}}}\right] \\
& =\mathrm{E}\left[\mathrm{e}^{\mu_{n}^{2} / 2 Z_{s_{n}}-u\left(\alpha_{n-1}^{\prime}, s_{n-1}-s_{n}\right) Z_{s_{n}}}\right] \mathrm{e}^{-2 \beta \sum_{i=1}^{n-1} \int_{0}^{s_{i}-s_{i+1}} u\left(\alpha_{i}^{\prime}, t\right) d t}+o(1) \\
& =\mathrm{E}\left[\mathrm{e}^{\mu_{n}^{2} / 2 Z_{s_{n}}+\alpha_{n-1}^{\prime} Z_{s_{n-1}}}\right] \mathrm{e}^{-2 \beta \sum_{i=1}^{n-2} \int_{0}^{s_{i}-s_{i+1}} u\left(\alpha_{i}^{\prime}, t\right) d t}+o(1) \\
& =\cdots \\
& \stackrel{r \downarrow 0}{\longrightarrow} \mathrm{E}\left[\mathrm{e}^{\sum_{i=1}^{n} \mu_{i}^{2} / 2 Z_{s_{i}}}\right]
\end{aligned}
$$

where we use the conditional on $Z_{s_{n}}$ in the second equation.

An application of Mukherjea et al [9] will give the result for any $\mu_{i} \geq 0$. The result thus follows.

\section{Acknowledgement}

H. Bi was supported by the Fundamental Research Funds for the Central Universities in UIBE (16QN04)

\section{References}

[1]. H. Bi and J.-F. Delmas. Total length of the genealogical tree for quadratic stationary continuous-state branching processes. Ann. Inst. H. Poincare Probab. Statist., 52(3):1321-1350, 2016.

[2]. Y.-T. Chen and J.-F. Delmas. Smaller population size at the MRCA time for stationary branching processes.Ann. Probab., 40(5):2034-2068, 2012.

[3]. T. Duquesne and J.-F. Le Gall. Random trees, Levy processes and spatial branching processes, volume 281. Asterisque, 2002.

[4]. M. Ji rina. Stochastic branching processes with continuous state space. Czechoslovak Mathematical Journal, 8(2):292-313, 1958.

[5]. K. Kawazu and S. Watanabe. Branching processes with immigration and related limit theorems. Theory of Probability \& Its Applications, 16(1):36-54, 1971.

[6]. J.-F. Le Gall. Ito's excursion theory and random trees. Stochastic Process. Appl., 120(5):721-749, 2010.

[7]. Z. Li. Measure-valued branching Markov processes. Springer Science \& Business Media, 2010.

[8]. Z. Li. Continuous-state branching processes. arXiv preprint arXiv:1202.3223, 2012

[9]. A. Mukherjea, M. Rao, and S. Suen. A note on moment generating functions. Stoch. Prob. Letters, 76(11):1185-1189, 2006. 